\title{
Simulations of (MC)AO for a 100m telescope
}

\author{
M. Le Louarn, Ch. Vérinaud, N. Yaitskova, V. Korkiakoski, E. Fedrigo, N. Hubin
}

\begin{abstract}
In this paper, we summarize the analysis made on various Adaptive Optics (AO) modes (including ShackHartmann (SH) and Pyramid Wavefront Sensors (PWS)) for the OverWhelmingly Large telescope (OWL). We will show some early results of the performance to be expected with a first generation AO system, working in the infrared.

Several telescope diameters were considered to see the variations as a function of telescope diameter. This is also compatible with the concept of "grow a telescope" where the telescope diameter of OWL grows from 60m to $100 \mathrm{~m}$ as a function of time.

In a first phase, to simplify the problem, the effects of the central obstruction were neglected. However, for the Shack-Hartmann ( $\mathrm{SH}$ ) simulations, additional simulations were carried out with a simulated OWL pupil, including segmentation errors.

We show some early results for a ground-layer correction system, working with three natural guide stars (NGSs) and a single deformable mirror (DM). An MCAO system based on 2 DMs, 3 NGSs is also investigated. For the last two systems, our results are found to be in very good agreement with Cibola, an analytical AO modeling tool. We show that some outer scale of turbulence effects improve slightly the correction quality when going from a telescope diameter of $10 \mathrm{~m}$ to $100 \mathrm{~m}$.
\end{abstract}

\section{INTRODUCTION}

Adaptive optics (AO) is a key component of all Extremely Large Telescope (ELT) designs. In this paper, we explore various AO systems, which are possible candidates for the ESO Overwhelmingly Large Telescope, OWL project. All systems are based on components which are likely to be available within the next ten years. They are "reasonable" extrapolations of current technology.

In the first section, we investigate the performance of a Single Conjugate AO system, based on a ShackHartmann sensor, and especially we show how performance evolves as a function of guide star magnitude. Then, the same study is carried out for the same system, but with a Pyramid wavefront sensor. The two systems are compared. Then we show some results of combining the AO system Point Spread Function (PSF) with the pupil shape and segmentation pattern of OWL. In the following section, we show some results on a ground layer AO system for OWL, based on 3 NGSs, providing a corrected FOV of 6'. Section 6 shows a study of the performance of an MCAO system, based also on 3 NGSs and 2 DMs, providing a corrected FOV of 2'. Finally, we present our conclusions.

\section{SHACK-HARTMANN SENSOR BASED SYSTEM SINGLE CONJUGATE AO}

We have studied the performance of a single conjugate AO system (SCAO) for OWL. The goal is to achieve diffraction limited imaging in the near infrared (K-Band) with technology close what is available today. We use a single deformable mirror and a single on-axis natural guide star. The parameters used for these single conjugate AO simulations are summarized in Table 2. We have also included a $10 \mathrm{~m}$ AO system, to better compare the evaluation of performance when going from current AO systems to AO for ELTs.

We can see that even for the $100 \mathrm{~m}$ case, only $\sim 8000$ actuators are required. This is compatible with an extrapolation of the adaptive secondary technology used on the MMT and LBT, assuming the current OWL optical design ( $2.5 \mathrm{~m}$ adaptive mirror). In the following, we investigate the performance as a function of the guide star magnitude of the SCAO system. 
Table 1. Parameters used in the SH simulation

\begin{tabular}{|l|l|l|l|}
\hline Parameter & Value OWL 100m & Value OWL 60m & Value $10 \mathrm{~m}$ \\
\hline Subapertures (linear) & 100 & 60 & 10 \\
Subaperture size (m) & 1 & 1 & 1 \\
Total slopes & 15592 & 5592 & 152 \\
CCD pixels / subap & $4 \mathrm{x} 4$ & $4 \mathrm{x} 4$ & $4 \mathrm{x} 4$ \\
RON / pixel (e) & 3 & 3 & 3 \\
Frame rate (kHz) & 1 & 1 & 1 \\
FOV per sub-aperture (as) & 2.4 & 2.4 & 2.4 \\
WFS wavelength ( $\mu \mathrm{m})$ & $0.7 \mu \mathrm{m}$ & 2987 & $0.7 \mu \mathrm{m}$ \\
\hline Total actuators & 8109 & 1 & 107 \\
Temporal delay (frames) & 1 & 1000 & 1 \\
Number of iterations & 1000 & 1 & 1000 \\
Simulation time (s) & 1 & 0.69 & 1 \\
\hline Seeing (at 0.5 $\mu \mathrm{m}$, in as) & 0.69 & 4.1 & 0.69 \\
Correlation time (at 0.5 $\mu \mathrm{m}$, in ms) & 4.1 & 26 & 4.1 \\
Outer scale of turbulence $(\mathrm{m})$ & 26 & 26 \\
\hline
\end{tabular}

\subsection{Centroiding algorithm and noise propagation}

Several ideas have been suggested to improve the performance of the Shack-Hartmann sensor at faint fluxes:

- Thresholding (for example $\left.{ }^{1}\right)$.

- Weighting the pixels according to some metric of Signal to Noise ratio $\left({ }^{2}\right)$.

- Weighting the pixels according to the mean value of the image $\left({ }^{3}\right)$.

- Hierarchical Shack-Hartmann sensor $\left({ }^{4}\right)$.

For our simulations, we used the simple approach proposed by, ${ }^{2}$ and took the 1.5 power of each pixel value before computing the centroid value. This allowed a slight improvement of the performance at all photon fluxes.

Noise propagation in a Shack-Hartmann sensor has been shown (see for example ${ }^{5}$ ) to be proportional to the logarithm of the linear number of sub-apertures across the telescope pupil $N$, if the sub-aperture size remains constant:

$$
\sigma^{2} \propto \ln N
$$

where $\sigma^{2}$ is the variance of the wavefront due to the noise propagation.

In our case, between the $10 \mathrm{~m}$ case and the $100 \mathrm{~m}$, the variance due to noise propagation should be multiplied by 2 . We will see the effects of this in the next section.

\subsection{Performance of the AO system}

The performance of the different systems was evaluated with different fluxes at the wavefront sensor. The centroiding algorithm used was the one described in the previous section.

We can see (Fig. 1, left) that between the $10 \mathrm{~m}$ and $100 \mathrm{~m}$ curve, a factor of about 2.2 more photons is required to achieve the same performance (here measured as the number of photons required to achieve a $30 \%$ Strehl ratio - half of the full flux performance). This is compatible with the increased noise propagation term.

Please note that all the performance estimates were done with a $1 \mathrm{kHz}$ frame rate and one frame pure delay. A more realistic 2 frames delay would reduce the performance. To improve the limiting magnitude, it is of course 

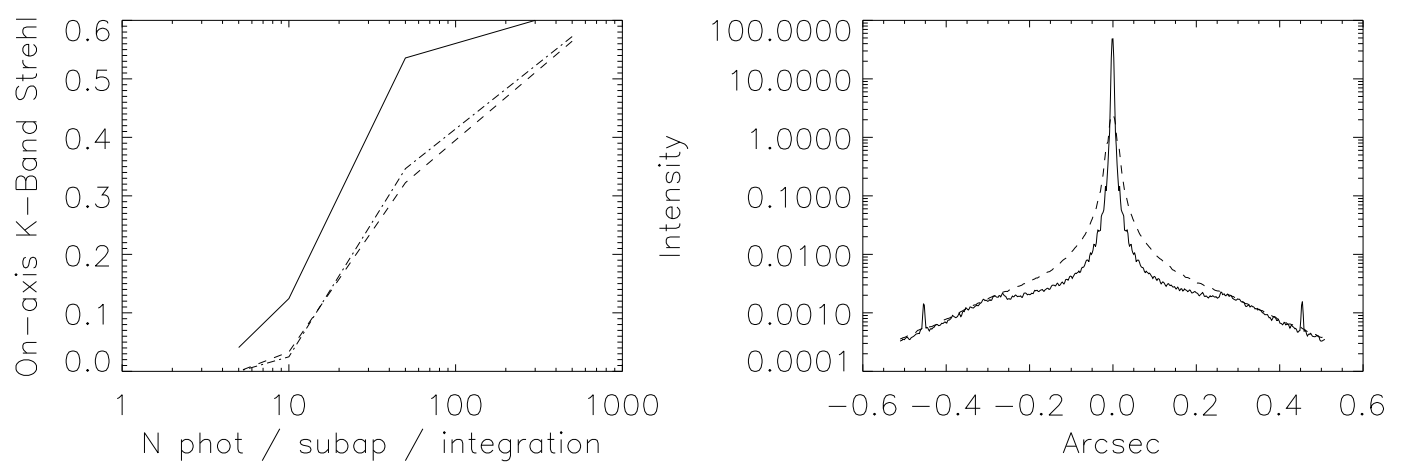

Figure 1. Left, performance (K-band) vs. number of photons per sub-aperture per integration time. The solid line is for the $10 \mathrm{~m}$ telescope, dash-dot for the $60 \mathrm{~m}$ case and dashed for the $100 \mathrm{~m}$ case. Note that all the points were drawn with a frame rate of $1 \mathrm{kHz}$, so they do not necessarily reflect a true limiting magnitude. Right, PSF (K-band) for the SH-WFS obtained with 500 photons / frame (solid) and $10 \mathrm{ph} /$ frame (dash). The Strehl ratio (at K band) is $56 \%$ and $3.3 \%$ respectively for 500 and 10 photons.

also possible to reduce the bandwidth to increase the integration time. This was not done here, since the goal was more to see the effect of the increased number of sub-apertures and telescope diameter.

Also, one can see that even the $10 \mathrm{~m}$ telescope performance is not very good compared to the current SCAO systems like NAOS. This is due to the lack of optimization of the frame rate to increase the number of collected photons for faint objects. However, this was done consistently for all telescope diameters. Also, it has been shown $\left({ }^{6}\right)$ that significant gains in limiting magnitude are obtained by modal optimization, windowing of the WFS detector, binning of the WFS CCD, thresholding etc. None of these were used on the SCAO simulation presented here. Our point is however to show that because of the $\mathrm{SH}$ noise propagation, some loss of performance is to be expected in terms of limiting magnitude on AO systems for ELTs.

\subsection{PSF for the $100 \mathrm{~m}$}

The effect on the PSF of the Strehl reduction can be seen in Fig. 1 (Right). On the high (56\%) Strehl PSF, the FWHM is $\sim 4.8$ mas. We can notice how the AO system starts to "clean" the seeing limited halo, by making a small hole around \pm 0.25 ". The spikes located around \pm 0.4 " are due to the influence functions used for the AO model, which are linear splines. These spikes disappear when a more realistic influence function model is adopted.

The lower Strehl PSF (Strehl at K-band of 3.3\%) has a FWHM of 10 mas. The PSF looks smooth, without many diffraction effects. We can notice how the basin due to AO correction has disappeared. This can be compared to the pyramid wavefront sensor behavior, described in the next section.

\section{PYRAMID WAVEFRONT SENSOR BASED SYSTEM}

In this section, we report on simulations of a $100 \mathrm{~m}$ ELT with an AO system based on a Pyramid Wavefront Sensor $\left({ }^{7}\right)$. No central obstruction is considered in a first stage. The algorithm used considers the PWS as a phase mask, and takes thus into account the interferences between the 4 pupils, that depend on the separation between them. In this paper we simulated a separation between the borders of the pupils of $30 \%$ the pupil diameter, like for example the pyramid sensor of the first-light AO system of LBT $\left({ }^{8}\right)$. A complete description of the algorithm can be found in. ${ }^{9}$ Both infrared $(2.2 \mu \mathrm{m})$ and visible $\mathrm{R}$ band $(0.7 \mu \mathrm{m})$ wavefront sensors are considered since different behaviors are expected depending on the phase residuals amplitude.

The system geometry and atmospheric parameters are the same as the $100 \mathrm{~m}$ case of table 1 . As well 1000 iterations equivalent to $1 s$ of integration time is considered. 
The simulation main characteristics are:

- For the Infrared sensor $(2.2 \mu \mathrm{m})$ no modulation is used neither on calibration nor during correction. Two simulations were run: one without read-out-noise and for high flux, and one with 100 photons and 17 electrons detector read-out-noise. The loop gain is 0.9 .

- For the visible sensor $(0.7 \mu \mathrm{m})$, the loop can be hardly closed when no modulation is used at calibration with poor performance. Therefore, calibration has been done with a $\pm 2 \lambda / D$ modulation angle, and the loop has been closed without modulation. Different fluxes from 100 to 5 photons per subaperture are considered and the CCD read-out-noise is 3 electrons. Note that the loop gain is here equal to 2.

\subsection{Strehl vs Magnitude}

Different fluxes from 100 to 5 photons per subaperture are considered and the read-out-noise is 3 electrons for the visible sensor. The bootstap and short exposure Strehl evolution is shown in Fig. 2. The results on fig. 3 (left) shows the result in long exposure Strehl in function of the mean number of photons per sub-aperture for the visible sensor (except for a high flux case with infrared sensor which is considered here as a reference for the highest Strehl achievable). It is remarkable that the loop can still be closed with very low flux (5 photons per sub-aperture (i.e 5 photons in all on 4 pixels) and 3 electrons read-out noise), with still a good Strehl.
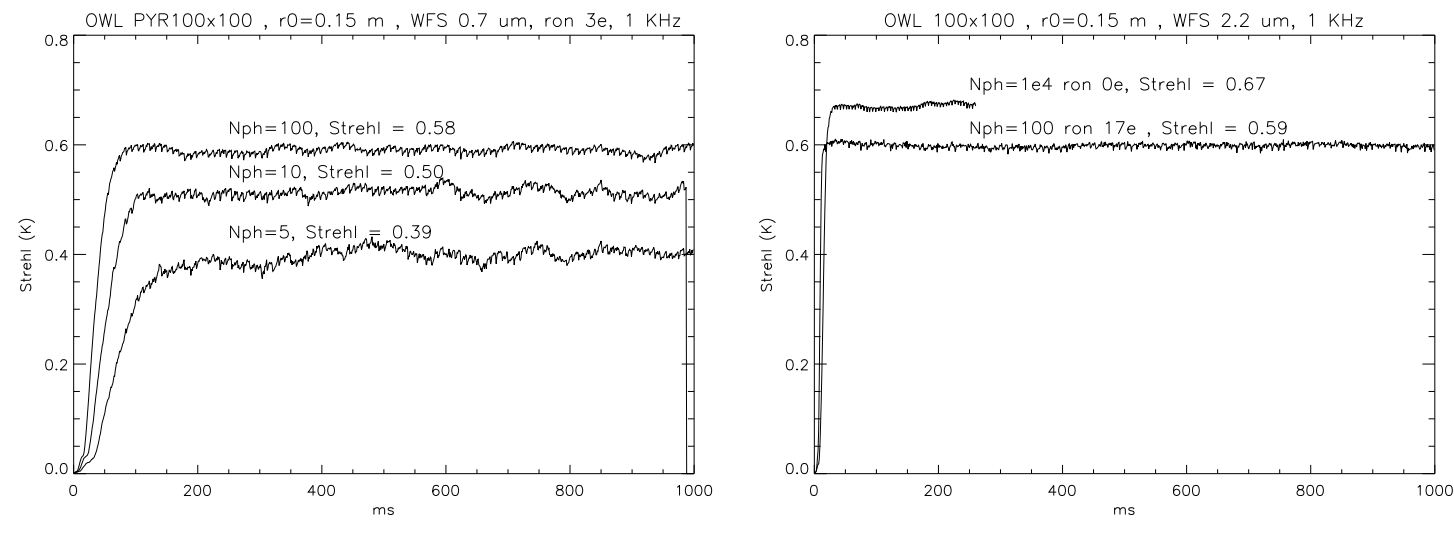

Figure 2. Strehl evolution for visible wavefront sensor (left) and for infrared wavefront sensor (right).

\subsection{PSFs}

The PSF corresponding to the different cases are shown in Fig. 4. Note that the $F W H M=4.5$ mas is almost the same for all cases. The effect of noise propagation being essentially a more or less uniform increase of the halo, but with very little perturbation on low orders that would decrease the resolution. Moreover the infrared sensor results, in the bright end regime, exhibit a deep hole in the PSF in agreement with the prediction of very low sensitivity to aliasing of the PWS when residuals are low $\left({ }^{10}\right)$

\subsection{Problem of reference measurements}

In any AO system, a reference measurement is usually subtracted from the actual measurements provided by the wavefront sensor. This reference measurement is the one given by the sensor in response to a flat wavefront, or corresponds to offsets yielding the flattest wavefront for the science detector (correction of non common path errors). We consider here no non-common path errors. However, because of the interferences between the 4 pupils on the detector of the PWS, the signal in response to a flat wavefront is never null, even with a perfectly aligned sensor. By default a reference is always computed for the actual configuration before starting the simulation and subtracted at each iteration. When no modulation is used this reference is by far not negligible and completely achromatic (see Fig. 3 (right), a measurement of 1 means a saturated measurement). This reference has been systematically subtracted for the infrared sensor simulations and this improved slightly the Strehl. For the visible 

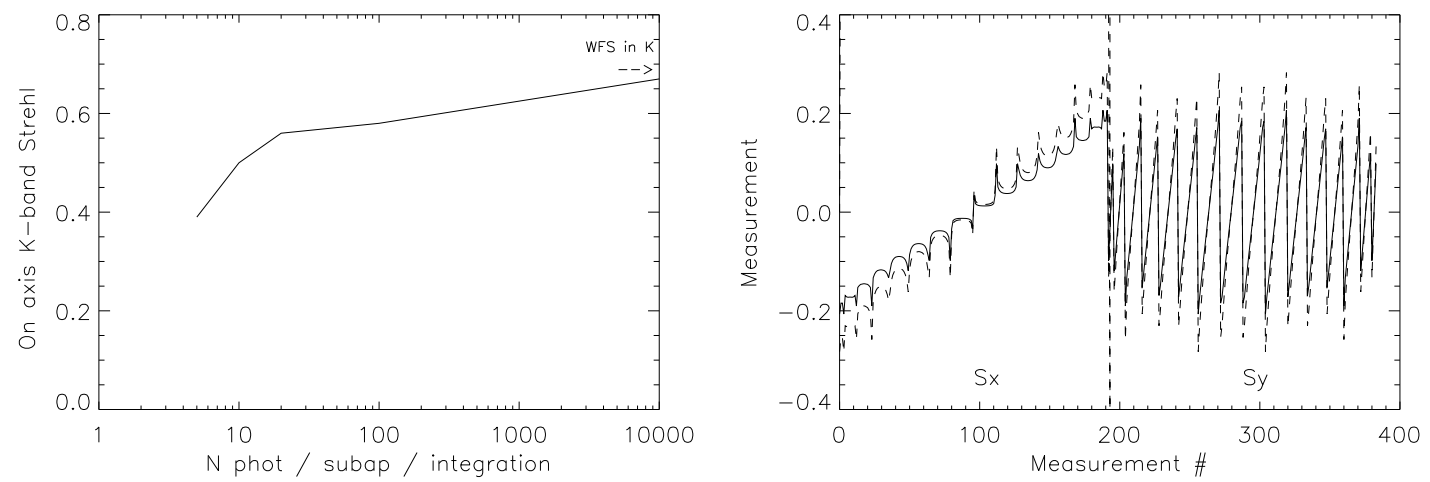

Figure 3. On the left, performance (K-band) vs. number of photons per sub-aperture per integration time for a $100 \mathrm{~m}$ telescope. Wavefront sensing is done at $0.7 \mu \mathrm{m}$ with 3 electron r.o.n., except for the 10000 photons case made at $2.2 \mu \mathrm{m}$ (as a reference for the highest Strehl achievable). Seeing: 0.69". On the right, measurement set due do interferences for a null incoming phase for a center-to-center pupil separation of 1.3 times the pupil size. No dynamic beam modulation.
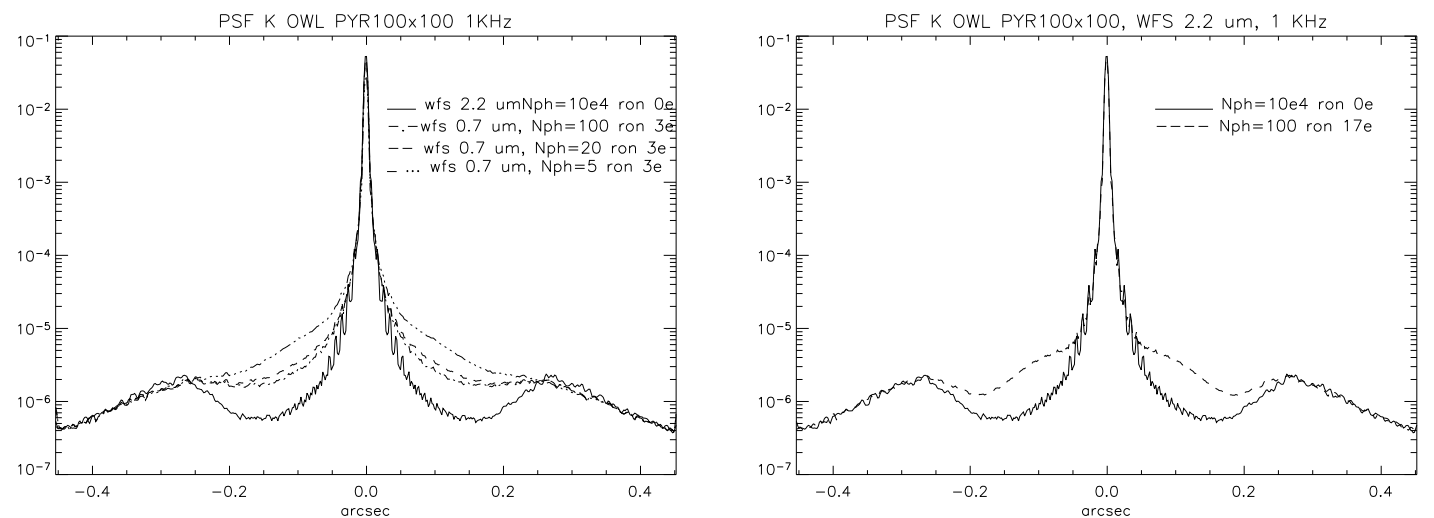

Figure 4. Left: PSF in K for different fluxes. FWHM is 4.5 mas. Right:PSF in $\mathrm{K}$ for an infrared wavefront sensor. Seeing: 0.69".

sensor, since the residuals are much larger, less inter-pupil interferences occur (contrary to the infrared one) and thus less or no offset measurement due to interferences are present. In this case, if the reference measurement is subtracted, the system is not able to converge. Thus no reference is subtracted for the simulations of the visible PWS. This peculiar behavior poses the problem of static aberrations or possible off-sets that one would like to add to the measurements in order to correct for non common path errors. So some remaining questions are: How does the PWS see static aberrations in the non linear regime ? Can modulation help in calibrating these static aberrations ? These problems seem to be similar to those encountered with quad-cells Shack-Hartmann sensors. Another issue for the PWS, is the question of on sky calibration for large deformable mirrors in an ELT for example. Using large modulation at calibration may be a solution, but the correspondence of the signals between large modulation and small or no modulation in closed loop may not be linear. A complete study is needed to investigate this important problem.

\subsection{Effect of central obstruction}

A more realistic simulation taking into account the fairly large central obstruction (35\%) of the OWL pupil has been attempted. The sub-pupil diameter is $80 \mathrm{~cm}$ and $r_{0}(0.5 \mu \mathrm{m})=0.2$. An infrared sensor is considered first because of the unnecessary modulation needed at calibration (as a first guess), since modulation requires a large 
computation time. The loop has been closed successfully, with a Strehl of $76 \%$. However, high noise propagation has been observed on actuators near the central obstruction (see Fig. 5) that may pollute significantly the residual halo. This has been identified as diffraction and interference effects occurring near the central obstruction and directed inside the geometrical pupil as it can be seen on Fig. 5. The use of modulation, at least at calibration should permit to damp this effect. This will be investigated in a future work since simulating modulation is rather time consuming, it has not been considered here.
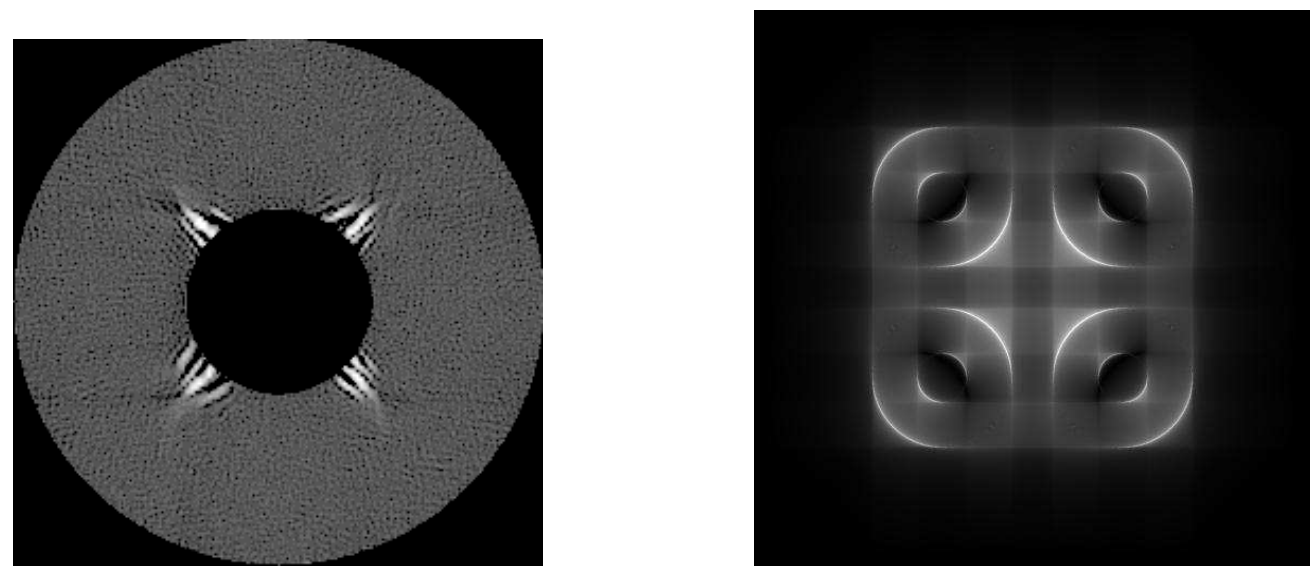

Figure 5. Left: Phase residuals with pyramid sensor and central obstruction. Maximum error amplitude near central obstruction is about $5 \mu \mathrm{m}$. Right: Diffraction effects at the pyramid sensor detector plane for a perfect wavefront and no modulation.

\section{EFFECT OF THE PUPIL AND SEGMENTATION}

The effects of segmentation and non-circular pupil shape were combined with the AO system residual phase to provide a first order evaluation of the PSF provided by OWL. In our present simulations, the segmentation effects and pupil shape have been added to the AO PSF, and therefore the segmentation effects are not taken into account in the AO closed loop. It is only at the PSF computation stage, at each iteration, that these elements are taken into account. A subsequent evolution of the AO simulation tool will be able to include these effects fully.

Only one realization of the co-phasing errors was used, since it is envisioned that during a one second exposure, as the one modeled here, the co-phasing errors will remain fairly stable.

The results can be seen in Fig. 7 (bottom right) and Fig. 6. A circular pupil mask (diameter 100m) was applied. The effect of the different segments being slightly out of phase can clearly be seen. The pupil shape shows the result of the combination of a segmented primary and secondary.

The rms phasing residual taken into account in this simulation is $42.0 \mathrm{~nm}$. Several interesting features can be seen in Fig. 7. Most of the features that can be easily seen are due to the hexagonal pupil shape and segmentation errors.

The complex structure of the PSF produced by the shape of the mirrors (without any phase errors) may be split into the four major structures according to the structures on the mirrors. Those are: (1) the secondary mirror support obscuration or the "spiders"; (2) gaps on the primary mirror, $14 \mathrm{~mm}$ in average; (3) gaps on the secondary mirror, $60.2 \mathrm{~mm}$ in the projection onto the plane of the primary mirror; and (4) "missing" segments, the segments which were removed from the whole shape of the primary as the part of the design. Each of the structures produces the particular pattern on the PSF:

- (1) The 6 branches of the "spider" produce the 6 narrow broken lines rotated at 90 degrees with respect to the direction of the branches. The width of the lines is inversely proportional to the distance between the 

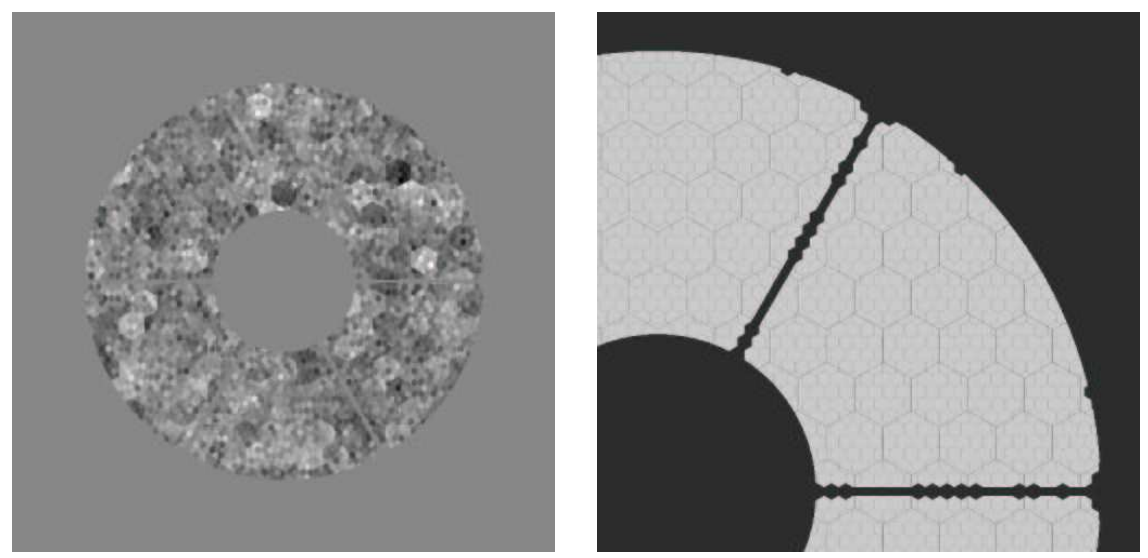

Figure 6. Co-phasing residuals in the OWL pupil (left) and part of the pupil shape (right) for the $100 \mathrm{~m}$ case.

two of the 6 branches along the same line and equals $0.0015 "$ (for the $0.5 \mu \mathrm{m}$ wavelength, here and below). The distance between the "holes" in the lines is the inverse of the branches width and equals 0.103 ".

- (2) Gaps on the primary mirror produce the regular pattern of the higher-order diffraction peaks following the hexagonal geometry. The period of the peaks is defined by the segments center-to-center separation. The period long $\mathrm{x}$ axis equals $0.074 "$, and the period along the y axis equals 0.13 ". The relative intensity of the peaks with respect to the intensity of the central is defined by the size of the gaps. For the 6 peaks closest to the center it is $310^{-5}$, which is in a good agreement with the theoretical predictions.

- (3) Gaps on the secondary mirror produce a similar pattern of the higher-order peaks, but on the smaller scale due to the projection of the mirror. There are many more peaks of this structure on the same 0.56 " field. The periods for the peaks along $\mathrm{x}$ and $\mathrm{y}$ axes are 0.018 " and 0.033 " correspondingly. The strongest diffraction is observed in the 6 directions corresponding to the direction along the line of the segments flat-to-flat conjunction. As predicted, the intensity of the peaks in these directions is independent on the distance to the center. It equals $510^{-6}$ for all peaks, except for the closest peaks, which are dominated by the rings of the Airy pattern.

- (4) The "missing" segments produce the additional flower-like shape with the short lines connecting the points of the higher-order peaks location. The structure has a hexagonal symmetry and the strongest diffraction is observed in the mention above 6 directions. The intensity of this pattern is comparable with the intensity of all other patterns, which is about $10^{-5}-10^{-6}$ of that for the central peak.

\section{GROUND LAYER CORRECTION}

In this section, we summarize our work on a ground layer correction system, working in the infrared. The system parameters are summarized in Table 2. The goal of this AO system would be to provide an improved seeing type of correction over a wide field in the near infra-red region (see an example for the VLT in ${ }^{11}$ ).

In this scheme, a single deformable mirror (conjugated to $0 \mathrm{~km}$ ) is used in conjunction with 3 natural guide stars, placed in a triangular configuration. The coordinates of the guide stars are: (180", 0), (-90", 156"), (-90", $\left.-156^{\prime \prime}\right)$, yielding a field of view of 6 ' in diameter. The performance of the system is evaluated only on-axis, for simplicity.

It can be seen that the deformable mirror used here is the same as the one used for the SCAO configuration. Therefore, this GLAO could be seen as an evolution of the SCAO system, which is made by adding more wavefront sensors. 


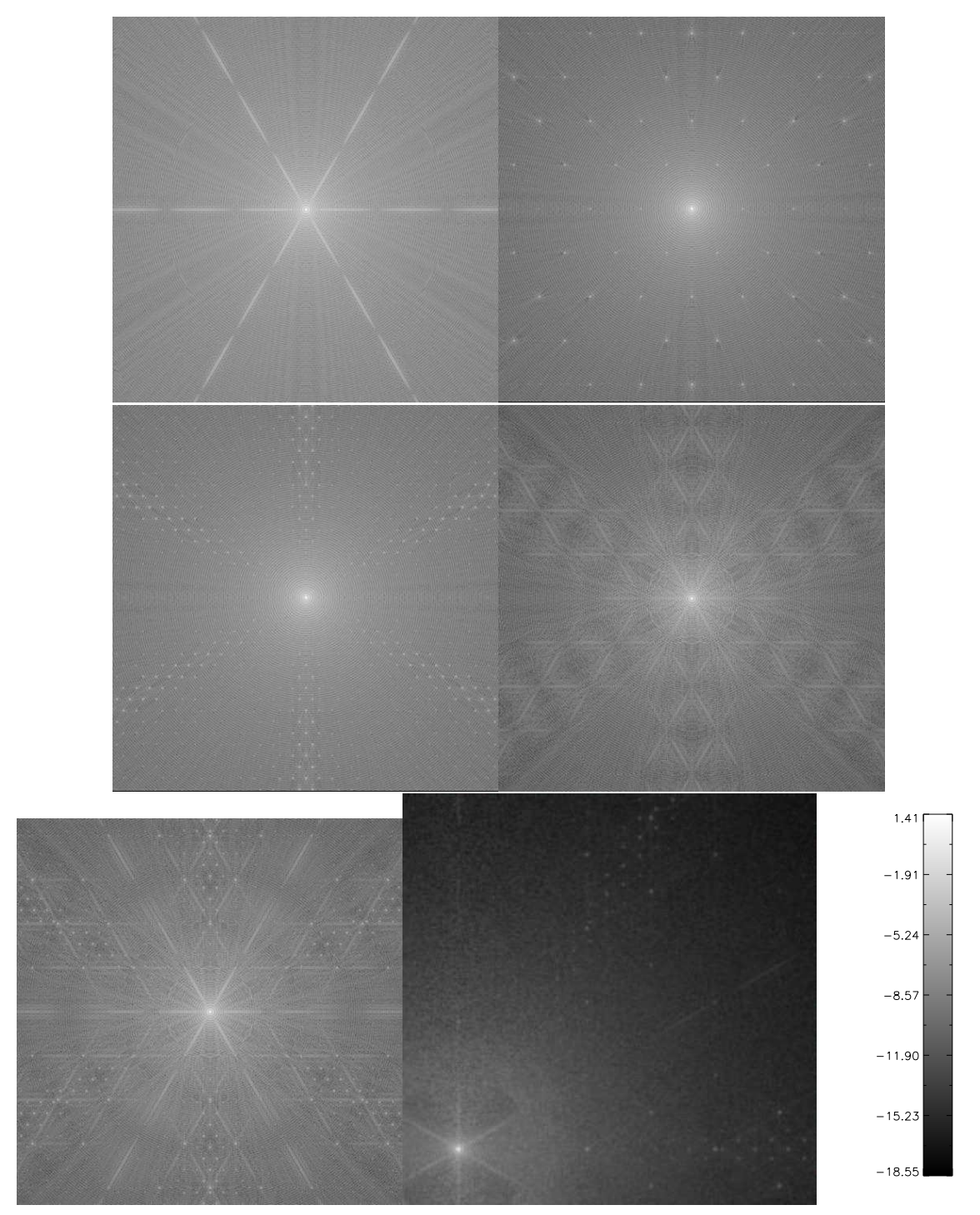

Figure 7. Effect of the PSF of the spiders (top left), gaps on the primary mirror (top right), gaps on the secondary mirror (middle left) and "missing" segments (middle right). The combined PSF is shown on the bottom left. For the aforementioned PSFs, the shown field is 0.56 " on the side. The bottom right image shows the combined effect of AO (note the corrected basin toward the central peak) and the full segmentation +spiders+pupil shape PSF. For the last PSF, the field is $1.8 "$

Figure 8 shows the evolution of the corrected PSF, when the telescope diameter is increased. We can see that as we go to larger diameters, the shape of the PSF changes dramatically. This effect, due to the outer scale of turbulence, was already analyzed for seeing limited PSFs by. ${ }^{12}$ Due to the size of the outer scale, even an uncorrected PSF can show a diffraction limited core. The Strehl is still extremely low, but turbulence doesn't have much power at the low spatial frequencies on ELTs, diffraction effects start to be seen. Here the situation is similar. Ground layer correction still provides only a seeing-limited type PSF. Here, we see that also GLAO benefits from this outer scale effect.

However, the energy in this peak is very small. Indeed, the Strehl ratio achieved with the 100m GLAO 
Table 2. Parameters used in the SH simulation

\begin{tabular}{|l|l|l|l|l|}
\hline Parameter - telescope & $\begin{array}{l}\text { Value OWL } \\
100 \mathrm{~m}\end{array}$ & $\begin{array}{l}\text { Value OWL } \\
60 \mathrm{~m}\end{array}$ & Value 30m & Value 10m \\
\hline Subapertures (linear) & 100 & 60 & 30 & 10 \\
Subaperture size (m) & 1 & 1 & 1 & 1 \\
Number of NGSs & 3 & 3 & 3 & 3 \\
Total slopes per WFS & 15592 & 5592 & 4128 & 152 \\
CCD pixels / subap & $8 \mathrm{x} 8$ & $8 \mathrm{x} 8$ & $8 \mathrm{x} 8$ & $8 \mathrm{x} 8$ \\
RON / pixel (e) & 3 & 3 & 3 & 3 \\
Frame rate (Hz) & 500 & 500 & 500 & 500 \\
FOV per sub-aperture (") & 1.4 & 1.4 & 1.4 & 1.4 \\
WFS wavelength ( $\mu \mathrm{m})$ & $0.5 \mu \mathrm{m}$ & $0.5 \mu \mathrm{m}$ & $0.5 \mu \mathrm{m}$ & $0.5 \mu \mathrm{m}$ \\
\hline Total actuators & 8109 & 2987 & 787 & 107 \\
Temporal delay (frames) & 1 & 1 & 1 & 1 \\
Number of iterations & 500 & 500 & 500 & 500 \\
Simulation time (s) & 0.5 & 0.5 & 0.5 & 0.5 \\
\hline Seeing (at 0.5 $\mu \mathrm{m}$, in as) & 0.69 & 0.69 & 0.69 & 0.69 \\
Correlation time (at 0.5 & 4.1 & 4.1 & 4.1 & 4.1 \\
$\mu \mathrm{m}$ in ms) & & & & 26 \\
Outer scale (m) & 26 & 26 & 26 & \\
\hline
\end{tabular}
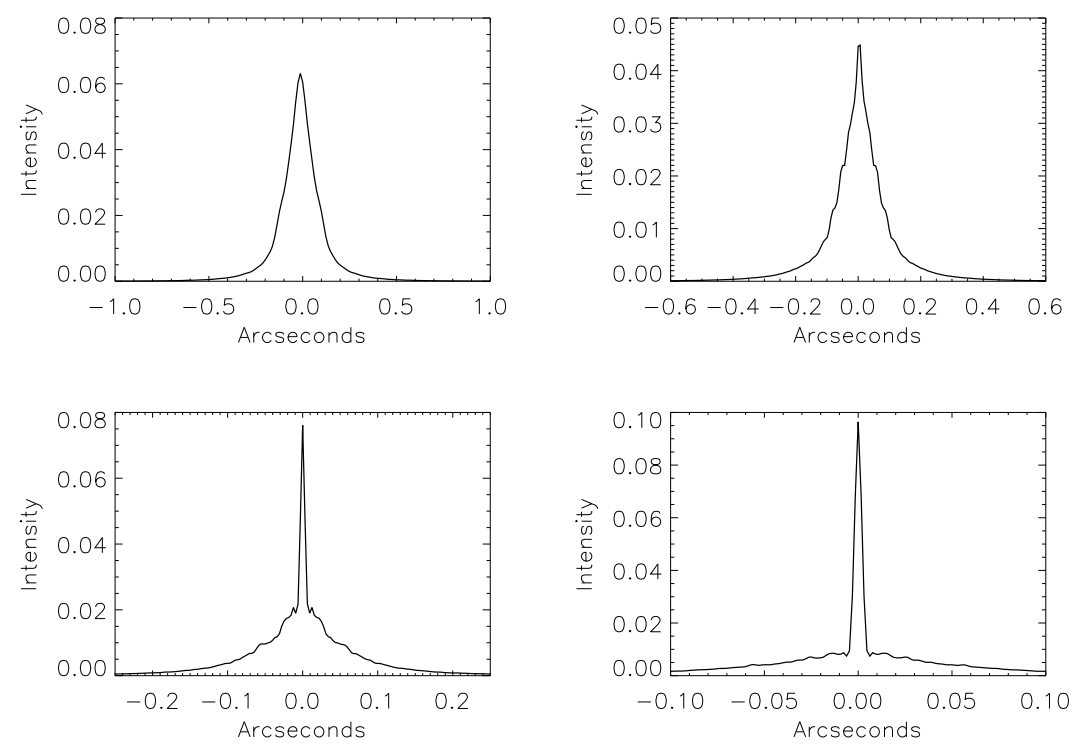

Figure 8. Corrected PSF using the 6' FOV GLAO system on 4 telescope diameters: from left to right and top to bottom: $10 \mathrm{~m}, 30 \mathrm{~m}, 60 \mathrm{~m}, 100 \mathrm{~m}$. Results are in K-Band, 0.69" seeing.

system is only $2.010^{-3}$. For the $60 \mathrm{~m}, 30 \mathrm{~m}$ and $10 \mathrm{~m}$ cases, it is respectively $3.110^{-3}, 8.510^{-3}, 4.510^{-2}$. It is interesting to note that in the GLAO system, the Strehl ratio still evolves like uncorrected seeing as a function of diameter.

A more interesting figure of merit for this AO system is the ensquared energy (EE) inside of a pixel of a given size. These are shown in Figure 9. On the Figure on the left, the EE as a function of pixel size is shown. 
We can indeed see that a very small fraction of the energy (less than a percent) is present in the high resolution peak. Most of the energy is still concentrated in the large seeing-like halo. This is confirmed by the plot on the right, which shows the energy in a 0.1 " pixel, which might be typical of the pixel size of an instrument using this GLAO system. We can see that this energy changes very little when the diameter is increased. We can also see that the uncorrected (seeing limited) PSF also benefits very slightly from the increase in telescope diameter. A comparison between our numerical code and Cibola, a Fourier space linear AO model $\left({ }^{13}\right)$ was made and is shown in Fig. 9 (right). The agreement is good, although some differences remain. The evolution of the EE in a pixel and the diameter is solely due to outer scale of turbulence in Cibola, as was verified by setting the value of L0 to $10 \mathrm{~km}$. This way, the EE in a pixel for the $100 \mathrm{~m}$ case comes back to the level of a 10m telescope. The difference between Cibola and our simulations can probably be attributed to the different reconstruction algorithm (MAP vs. minimum variance). The atmospheric model being quite optimistic allows the minimum variance algorithm (which has a complete knowledge of the atmospheric profile) to outperform the MAP approach (which in the particular case of GL-AO has no turbulence profile information, since all the profile is collapsed into a single DM). As we shall see in the following, a comparison with MCAO is much more accurate.

Please note that in these plots, we show the fraction of the flux in the pixel. The absolute value of this flux is of course much larger (for the same integration time) on the large telescopes.
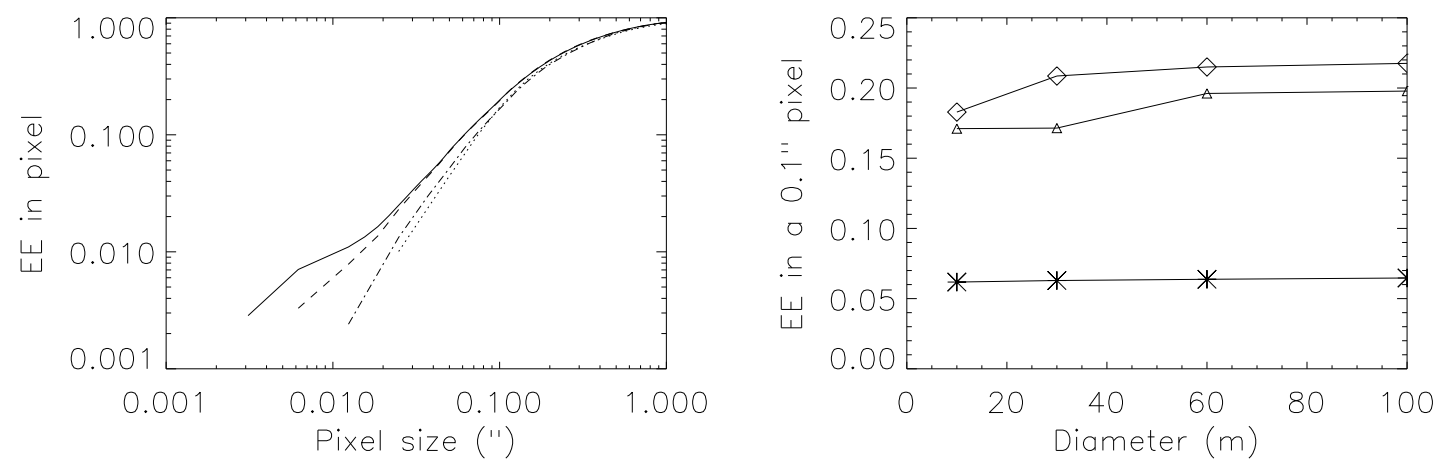

Figure 9. Left: Fraction of EE in a pixel as a function of pixel size (in ") for 4 telescope diameters: 10m (dots), 30m (dot-dash), 60m (dash), 100m (solid). Right: Fraction of EE in a 0.1" pixel, as a function of telescope diameter (triangles) and for uncorrected seeing (asterisks). The diamonds show the results obtained with Cibola, in closed loop. All results are in K-band, 0.69" seeing.

\section{MULTI-CONJUGATE AO (NGS-BASED)}

The next step in complexity is represented by an MCAO system, where another DM is added compared to the GLAO system. We placed this DM at an altitude of $7.0 \mathrm{~km}$ above the telescope. Three NGSs are used, but they are now placed in a constellation of 2' in diameter, which is also the size of the corrected field (defined by the high altitude deformable mirror). All NGSs are assumed to be bright. This system resembles somewhat the MAD (MCAO Demonstrator ${ }^{14}$ ), however the simulation conditions and precise parameters are different, so a direct comparison of the performance is not possible.

The result of these simulations is presented in Figure 10. The Strehl ratio (on-axis) is plotted as a function of telescope diameter. As we can see, a small increase in performance is obtained as the diameter increases. It is not possible to say if this effect is due to a better beam overlap for the $100 \mathrm{~m}$ case and outer scale effects. However, other effects, like generalized anisoplanatism $\left({ }^{15}\right)$, fitting and aliasing errors dominate the performance. Therefore the gain in performance is small. These simulation results were confirmed by running Cibola with the same parameters. We can see that for large diameters (where Cibola is the most valid, since it assumes an infinite aperture for some error sources), the agreement between the two simulations is remarkably good. 


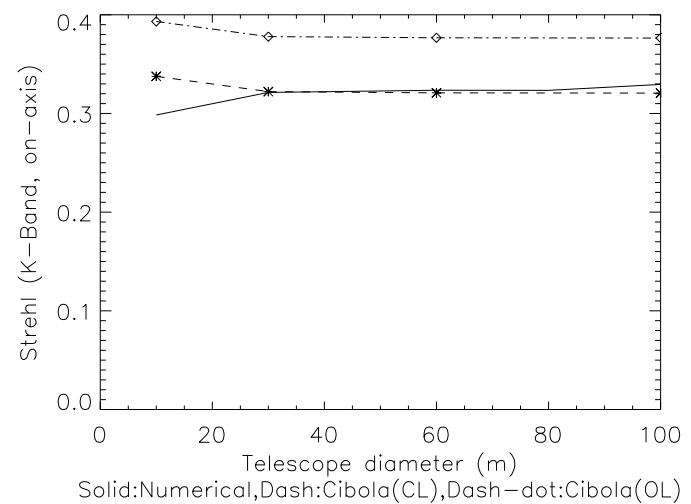

Figure 10. On-axis Strehl as a function of telescope diameter for the MCAO system, for our numerical simulation (solid line), Cibola with an open loop minimum variance reconstructor (diamonds) and Cibola with a filtered closed-loop minimum variance reconstructor (asterisks).

\section{CONCLUSION, FUTURE WORK}

There are different options which we should investigate to improve the performance at low flux for the ShackHartmann (and possibly Pyramid) systems:

- A better control matrix for the AO system (possibly based on minimum variance), which takes explicitly into account the closed loop operation of the system will be investigated.

- Optimized modal control should perform significantly better at low flux levels than the current control scheme, which relies on a simple integrator with one gain. Also other methods to increase the overall $\mathrm{SH}$ performance at faint fluxes shall be implemented.

- The hierarchical SH should provide better noise propagation characteristics than a single large SH and should be investigated.

- Other "centroid" calculation schemes (like that proposed by ${ }^{3}$ could also bring a gain in limiting magnitude.

- For the Pyramid sensor, a better understanding of diffraction effects is needed, for the central obstruction issue and also for segmentation which was not taken into account here.

Another improvement to these simulations will be the addition of segmentation / pupil shape in the AO code itself. Indeed, the secondary supports for example will reduce the illumination (or suppress illumination) to some sub-apertures. This could reduce the performance of the AO system.

The parameter space remains large also for the two other systems. In the GLAO, different number of guide stars, and guide star positions are to be investigated. Also, the stability as a function of field is important. Other wavefront sensing schemes (Layer-oriented, for example) will also be studied in the future. The same kind of studies will also be made for the MCAO case.

Obviously, many more simulations are required in the study of the AO systems for OWL. These are foreseen to be made in the framework of the Framework Program 6 - ELT design study, which is presented in more details in. ${ }^{16}$

\section{Acknowledgments}

The authors would like to thank Philippe Dierickx for discussions concerning OWL and the AO for OWL. Also, Brent Ellerbroek provided a significant improvement to this paper by providing and helping us with his Cibola simulation tool. 


\section{REFERENCES}

1. E. Gendron, Optimisation de la commande modale en optique adaptative: applications à l'astronomie. $\mathrm{PhD}$ thesis, Université D. Diderot, mar 1995.

2. M. Kasper, D. Looze, S. Hippler, R. Davies, and A. Glindemann, "Increasing the sensitivity of a shackhartmann sensor," in Proceedings of the Canterbury Conference on Wavefront sensing and its applications, Canterbury, 1999.

3. M. Nicolle, T. Fusco, V. Michau, and G. Rousset, "Increasing the sensitivity of a shack-hartmann sensor," in Advancements in Adaptive Optics, 5490, SPIE, Proc. SPIE, 2004.

4. B. Le Roux, J. Coyne, and R. Ragazzoni, "Hierarchical wave-front sensing," Astron. Astrophys. submitted, 2004.

5. F. J. Rigaut, J.-P. Veran, and O. Lai, "Analytical model for shack-hartmann-based adaptive optics systems," Proc. SPIE 3353, pp. 1038-1048, Sept. 1998.

6. T. Fusco, G. Rousset, D. Rabaud, E. Gendron, D. Mouillet, F. Lacombe, G. Zins, P.-Y. Madec, A.-M. Lagrange, J. Charton, D. Rouan, N. Hubin, and N. Ageorges, "Naos on-line characterization of turbulence parameters and adaptive optics performance," J. Opt. A.: Pure and applied optics 6, pp. 585-596, 2004.

7. R. Ragazzoni J. Mod. Opt. 43, p. 289, 1996.

8. S. Esposito, A. Tozzi, D. Ferruzzi, M. Carbillet, A. Riccardi, L. Fini, C. Verinaud, M. Accardo, G. Brusa, D. Gallieni, R. Biasi, C. Baffa, V. Biliotti, I. Foppiani, A. Puglisi, R. Ragazzoni, P. Ranfagni, P. Stefanini, P. Salinari, W. Seifert, and J. Storm, "First-light adaptive optics system for large binocular telescope," in Adaptive Optical System Technologies II, P. L. Wizinowich and D. Bonaccini, eds., Proc. SPIE 4839, SPIE, 2003.

9. C. Vérinaud, C. Arcidiacono, M. Carbillet, E. Diolaiti, R. Ragazzoni, E. Vernet-Viard, and S. Esposito, "Layer Oriented multi-conjugate adaptive optics systems: performance analysis by numerical simulations," in Adaptive Optical System Technologies II. Edited by Wizinowich, Peter L.; Bonaccini, Domenico. Proceedings of the SPIE, Volume 4839, pp. 524-535 (2003)., pp. 524-535, Feb. 2003.

10. C. Verinaud, "On the nature of the measurements provided by a pyramid wavefront sensor," Opt. Commun. 233, pp. 27-38, 2004.

11. M. Le Louarn and N. Hubin, "Wide-field adaptive optics for deep-field spectroscopy in the visible," $M N R A S$ 349, pp. 1009-1018, Apr. 2004.

12. R. Conan, M. Le Louarn, J. Braud, E. Fedrigo, and N. N. Hubin, "Results of AO simulations for ELTs," in Future Giant Telescopes. Edited by Angel, J. Roger P.; Gilmozzi, Roberto. Proceedings of the SPIE, Volume 4840, pp. 393-403 (2003)., pp. 393-403, Jan. 2003.

13. B. L. Ellerbroek, "Linear systems modeling of adaptive optics in the spatial frequency domain," J. Opt. Soc. Am. A submitted, 2004.

14. E. Marchetti, R. Brast, B. Delabre, R. Donaldson, E. Fedrigo, F. Franza, N. Hubin, J. Kolb, M. Le Louarn, J. Lizon, S. Oberti, R. Reiss, J. Santos, R. Ragazzoni, C. Arcidiacono, A. Baruffolo, E. Diolaiti, J. Farinato, and E. Vernet-Viard, "MAD status report," in Advancements in Adaptive Optics, Proc. SPIE 5490, 2004.

15. A. Tokovinin, M. Le Louarn, and M. Sarazin, "Isoplanatism in a multi-conjugate adaptive optics system," J. Opt. Soc. Am. A 17, pp. 1819-1827, 2000.

16. N. Hubin, E. Marchetti, J. Farinato, E. Fedrigo, M. Ferrari, T. Fusco, M. Le Louarn, R. Ragazzoni, and M. Tallon, "Status of the adaptive optics point designs for the European ELT design study," in Advancements in Adaptive Optics, Proc. SPIE 5490, 2004. 